\title{
Artificial Bee Colony-based General Adversarial Network for Liver Cancer Detection using CT Images
}

\author{
Rashid Khan \\ National Engineering Laboratory of Speech and Language Information Processing(NELSLIP) \\ Department of Electronic Engineering \& Information Science, \\ University of Science and Technology of China (USTC), Hefei, PR China. \\ khanrashidkk7@gmail.com
}

\begin{abstract}
Nowadays, liver cancer is the foremost cause of death and the Computed Tomography (CT) images are widely utilized for detecting liver cancer detection. However, the manual classification and detection of liver tumor based on the CT scan images is the challenging one. In this paper, the Artificial Bee Colony based General Adversarial Network (ABCbased GAN) is developed for detecting liver cancer using CT images. The developed model mainly consists of four stages, namely liver extraction, cancer lesion segmentation, feature extraction, and classification. Initially, the input liver CT images are fed to the liver extraction stage where the active contour method is employed for extracting the liver region from CT images. After that, the cancer lesion regions are segmented from the extracted liver images using the Markov Random Field (MRF). Once the cancer lesions are segmented, then, the feature extraction process is done based on statistical features, like mean, standard deviation, skewness, and kurtosis. Finally, the cancer classification is performed based on the extracted features using the GAN classifier where the ABC optimization algorithm is utilized to train the GAN classifier. Furthermore, the developed method achieved maximal accuracy of $95.16 \%$, maximal sensitivity of $91.88 \%$, and maximal specificity of $98.42 \%$.
\end{abstract}

Keywords: Liver Cancer, Markov Random Field, Malignant Tumor, Artificial Bee Colony Algorithm, General Adversarial Network.

\section{Introduction}

The liver presents below the diaphragm and enlarges from right to left upper division of the belly in the human body. The liver is a human organ, which has many tasks to create various chemicals required for the human body. In worldwide, liver cancer is one of the major causes of death that is increasing day-byday [1]. Primary liver cancer is otherwise called Hepatocellular Carcinoma (HCC), which is the third main donor of cancer-related deaths globally [6] [4]. In the clinical diagnosis classification, the prevention and treatment of liver cancer is the main focal point [7] [2]. The HCC and metastatic carcinomas are the familiar groups of liver cancer disease, and it is identified using a computer-aided diagnosis method [8] [2]. The separation of the cancerous segment using the segmentation technique is unavoidable for identifying cancer. The identification of infectious tissue is somewhat complex, because of the structural alternations in the liver by body shape, sex, age, and less contrast among the normal and infectious tissues. In the liver, the differentiation of HCC from metastatic carcinomas is complex due to the appearance of these two tumors [2]. Although the texture is tough, edges are unequal, and also surface abnormality is considered through the small or huge nodules in the HCC liver cancer. Hence, the identification of liver lesions offers a significant source for successive treatment and clinical preparation [9] [5]. Traditionally, medical image methods, like Magnetic resonance Imaging (MRI) and Computed Tomography (CT) images are utilized for detecting and diagnosing liver cancer [24, 25]. Furthermore, image fusion is the process of combining single composite images and multiple input images, and it is utilized in biomedical image processing, such as tumor detection, quality of the image, and so on [4]. Normally, there are two types of categorization are available, namely malignant and benign tumors. The benign tumor is non-cancerous, and further, it multiplies in the body. Subsequently, this type of tumor is cured easily and less chance to spread to other parts of the body. Another type of malignant tumor is very 
risky that spreads easily to other parts of the body. This malignant tumor is mainly created by the irregular cells, which are unbalanced, and their development is uneven [3].

The CT images are widely utilized for detecting the cancerous tissues precisely [10] [1]. At present, for the detection of liver cancer, contrast-enhanced triple-phase CT images are commonly utilized. The CT images are obtained by injecting the iodinated contrast agent into the patient, and the image is obtained at three various time intervals. Three stages are followed during the CT image acquisition, such as the arterial stage, Portal Venous (PV) stage, and the hepatic venous phase. Moreover, the images obtained from the CT scan are utilized for detecting the cancerous region of the liver [2]. Even though, the CT images represent the cancerous region of the liver, it can be enhanced with respect to precision, high resolution, and so on. Therefore, the enhanced CT images offer better visual analysis for liver cancer detection [4]. In the image processing techniques, the Computer-Aided Diagnosis (CAD) is widely utilized for the detection of liver cancer [11] [1]. Mainly, three methods are utilized to detect liver cancer, namely blood test, image test, and biopsy. Watershed and thresholding techniques are developed for liver cancer detection [12] [3]. In [13] [3], fuzzy C-means clustering and gray level co-occurrence matrix is applied for the liver tumor classification in mammography images. Besides, an automated liver cancer detection technique is developed based on the MRF method [14] [3]. An effective optimized technique was developed using Support Vector Machine (SVM) and instance optimization for liver cancer detection [15] [1]. Furthermore, the edge-based distance regularized level-set analysis approach is developed for liver cancer classification [16] [1]. This method efficiently segmented the calculi, tumor, cyst, and normal liver in the CT images. Additionally, the multi-channel fully convolutional network method is developed for detecting the liver tumor accurately [17] [1].

The main intention of the research is liver cancer detection in CT images using ABC-based GAN. Initially, the liver CT image database is given to the liver extraction phase. In the liver extraction phase, the active contour method is applied for extracting the liver regions from CT images. After that, the cancer lesion regions are segmented using the MRF method. The segmented cancer lesions are fed into the features extraction phase for the cancer classification. In the feature extraction stage, the significant statistical features, such as mean, standard deviation, kurtosis, and skewness are extracted. Once the feature extraction is done, the classification of the cancer stage is executed using a GAN classifier for detecting liver cancer from $\mathrm{CT}$ images. Also, the ABC optimization algorithm is utilized to train the developed GAN classifier.

The major contribution of the paper is the Proposed Artificial Bee Colony-based General Adversarial Network for the liver cancer classification. The characteristic features of $\mathrm{ABC}$ achieve the best detection result through the optimal fitness value.

The paper is organized as follows: section 2 elaborates the literature review of the existing liver cancer detection methods using CT images. Section 3 discussed the proposed ABC-based GAN for detecting liver cancer using CT images. Section 4 elaborates the results and discussion of the proposed method, and finally, section 5 concludes the paper.

\section{Literature Survey}

This sub-section elaborates the existing approaches of liver cancer detection as follows. Das A et al. [1] developed deep learning-based watershed management and Gaussian mixture method for liver cancer detection using CT images. In this method, the liver images were separated through the watershed segmentation method. After that, cancer affected regions were identified using the gaussian mixture model technique. Once the tumor segmentation was done from the segmented regions, different texture features were extracted. Furthermore, the segmented features were subjected to the Deep Neural Network (DNN) classifier for classifying the types of liver cancer. The three types of liver cancer, such as metastatic carcinoma, metastatic carcinoma, and hemangioma was considered. Das A et al. [2] introduced a modified fuzzy clustering technique and decision tree classifier for detecting liver cancer in CT images. Here, the spatial fuzzy clustering and adaptive thresholding were combined for identifying the cancer regions in CT scan images. At first, the liver regions were separated from other parts in CT images through the adaptive thresholding. Then, cancer affected lesions were separated from the liver using the spatial fuzzy clustering. Moreover, the required features were extracted for classifying liver cancer. In this method, the two types of liver cancer, metastatic carcinoma, and hepatocellular carcinoma were identified using the decision tree classifiers and the multilayer perceptron. This method utilized a 10 -fold cross-validation approach for evaluating the performance of the classifier. Hemalatha $\mathrm{V}$ and Sundar C [3] presented the soft optimizing approach for automatic liver cancer detection in CT images. In this method, scanning, filtering, segmentation, feature extraction processes were performed for liver cancer detection. Initially, the filtering process was applied for removing the noises in CT images and smoothing the edges. Then, the segmentation process was executed for extracting the required region for 
classification. After that, the gray level matrix and co-occurrence matrix was implemented for the feature extraction. These matrices were used to classify the two types of liver cancer, such as malignant and benign. Also, a feed forward neural network was utilized for classifying and detecting liver cancer. On the other hand, the Artificial Neural Network (ANN) was also employed for separating the two types of cancers. Lakshmi Priya B et al. [4] developed a multi-slice fusion structure for liver cancer detection in CT images. In this method, anisotropic diffusion filtering and multi-slice image fusion were combined with a non-subsampled contourlet field for improving edge information of CT images. The nearby two image slices were decomposed through the non-subsampled contourlet, and also the high frequency and low frequency coefficients were combined through the sum-modified Laplacian operators and phase congruency. Also, the images were processed through the anisotropic diffusion for strengthening edge information. After that, the edge enhanced images were obtained through the non-subsampled contourlet reconstruction.

\section{Proposed Artificial Bee Colony Optimization Algorithm for Liver Cancer Classification}

This section explains the developed Artificial Bee Colony algorithm for liver cancer detection in CT images. The proposed approach includes the following four steps, namely liver extraction, cancer lesion segmentation, feature extraction, and liver cancer classification. Initially, the database of liver CT images is fed into the liver extraction phase. In the liver extraction stage, the liver images are extracted from CT images using the active contour method [18]. After that, the cancer lesions are segmented in the cancer lesion segmentation phase through the MRF method [19]. Once the cancer lesions are segmented then, the statistical feature [20] extraction is performed. Finally, the liver cancer classification is performed by using the GAN [21] in which the ABC algorithm [22] is utilized to train the GAN method. Fig. 1 represents the schematic view of a developed ABC-based GAN for liver cancer detection in CT images.

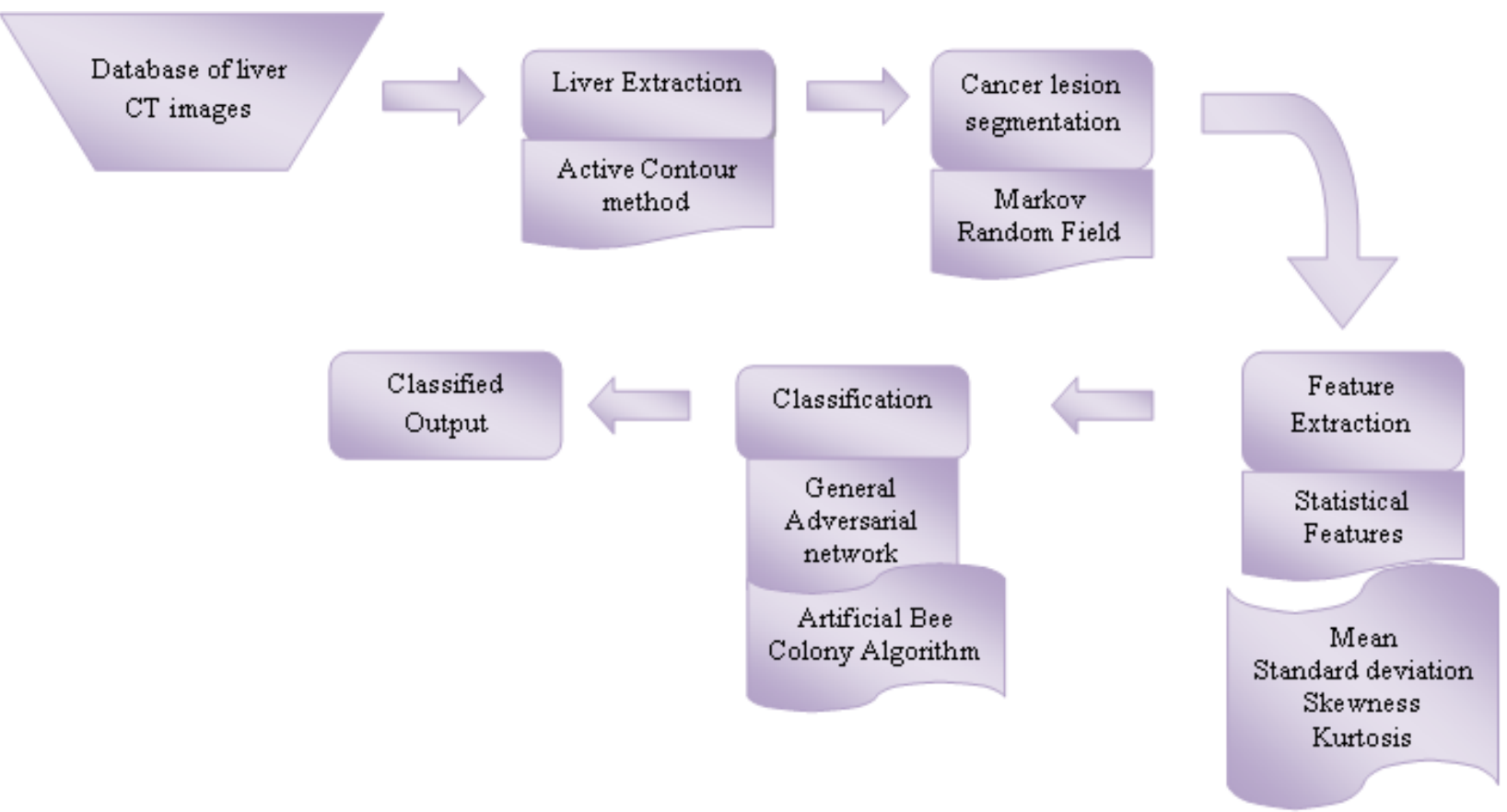

Fig.1. Schematic view of a developed artificial bee colony-based general adversarial network for liver cancer detection in CT images

\subsection{Input Acquisition}

The input images are gathered from the liver CT image database, and it is utilized to detect the liver cancer process. Let us assume a dataset $\mathrm{L}$ with $\mathrm{l}$ cancer images, and it is denoted as below

$$
\mathrm{L}=\left\{\mathrm{B}_{1}, \mathrm{~B}_{2}, \ldots, \mathrm{B}_{\mathrm{z}}, \ldots \mathrm{B}_{\mathrm{n}}\right\}
$$

where, $L$ is a database, $B_{n}$ represents total input images, $B_{z}$ illustrates the image, which is located in $\mathrm{z}^{\text {th }}$ index, and the number of images is denoted as $n$. The image $\mathrm{B}_{\mathrm{n}}$ is selected for the next preprocessing stage. 


\subsection{Liver Extraction using Active Contour}

The image $B_{n}$ is selected as the input to the liver extraction stage. Here, the active contour method [18] is employed for liver extraction from CT images. The active contour method increases the capture regions around the features of interest. The active contour method is employed for smoothing the structure of the boundary. The active contour method is utilized for interactive specifications of image contours. Moreover, it offers the combined relation of visual problems, such as identification of lines, subjective contours and edges, stereo matching, and motion tracking. In active contour method, the interactive interpretation is utilized, in which the user imposed limitation force controls the snakes for near features of interest. The outcome of the liver extraction phase is denoted as $\mathrm{B}_{c}$, and it is given to the cancer lesion segmentation stage.

\subsection{Cancer Lesion Segmentation based on Markov Random Filed}

The liver extracted image $B_{c}$ is taken as the input for the cancer lesion segmentation process for detecting cancer lesion regions. Segmentation is a process of separating the images into different segments, like image objects or pixels. Here, the MRF method [19] is performed for segmenting the cancer lesion regions from the liver CT images. MRF is commonly utilized in medical image segmentation since this method can easily represent the intensity in homogeneities occurring in medical images. The MRF method encodes information about the stability of neighboring image areas. MRF methods provide a better result with respect to the spatial relationship among neighboring pixels, and its computation overhead is less than the other segmentation methods. The major goal of the MRF method is that the pixel is more probable to a particular tissue type if the neighboring pixels are also of a similar kind. The segmented cancer lesion region output is denoted as $\mathrm{B}_{\mathrm{s}}$, which are further forwarded to the feature extraction phase for further processing.

\subsection{Feature Extraction using Statistical Features}

The segmented output $\mathrm{B}_{\mathrm{s}}$ is taken as the input for feature extraction in which the segmented features [20], such as skewness, kurtosis, mean and standard deviation are extracted from the segmented image. Here, the feature extraction process is applied for extracting valuable data from the image. The feature extraction process is very necessary for detecting liver cancer precisely. The statistical feature extraction guarantees the efficiency of liver cancer detection. The statistical features extracted from $\mathrm{B}_{\mathrm{s}}$ are given below:

a) Mean: Mean is estimated by calculating the average of pixels enclosed in an image, and it is represented as,

$$
\mathrm{C}_{1}=\frac{1}{\left|\mathrm{~s}\left(\mathrm{P}_{\mathrm{b}}\right)\right|} \times \sum_{\mathrm{b}=1}^{\left|\mathrm{s}\left(\mathrm{P}_{\mathrm{b}}\right)\right|} \mathrm{s}\left(\mathrm{P}_{\mathrm{b}}\right)
$$

where, $b$ denotes the total segments, $s\left(P_{b}\right)$ represents pixel values of every segment, and also total pixels enclosed in a segment is illustrated by $\left|\mathrm{s}\left(\mathrm{P}_{\mathrm{b}}\right)\right|$, and $\mathrm{C}_{1}$ represents the mean.

b) Standard Deviation: The square root of the variance is represented by the standard deviation, and it is denoted as $\mathrm{C}_{2}$.

c) Skewness: It illustrates the object shape with respect to arithmetical value. It also signifies the relative symmetry, and the skewness is represented as $\mathrm{C}_{3}$.

c) Kurtosis: The kurtosis defines the symmetry, and it describes the sharpness of peak, and the kurtosis is indicated as $\mathrm{C}_{4}$.

At last, the statistical features extracted from the segmented result are denoted as $\mathrm{C}_{\mathrm{t}}=\left\{\mathrm{C}_{1}, \mathrm{C}_{2}, \mathrm{C}_{3}, \mathrm{C}_{4}\right\}$ with dimension of $[4 \times 1]$, correspondingly.

\subsection{General Adversarial Network for Liver Cancer Detection}

The extracted features $\mathrm{C}_{\mathrm{t}}$ from cancer lesion segmentation images are fed to GAN[22] for detecting liver cancer. GAN is a deep learning classifier, which obtains the precise level of access to liver cancer detection. GAN is generally utilized for creating more exact prediction output in a complex circumstance. GAN is included with two various elements, such as a generator and a discriminator. The generator aims to puzzle the discriminator by a generation of possible data, while the discriminator is utilized for detecting the fake data from a real set of data. GAN records the data samples from the prior distribution 
with data samples of other distribution. Although, the mapping process is obtained by using a generator component to accurately detect liver cancer. Fig. 2 shows the architecture of GAN.

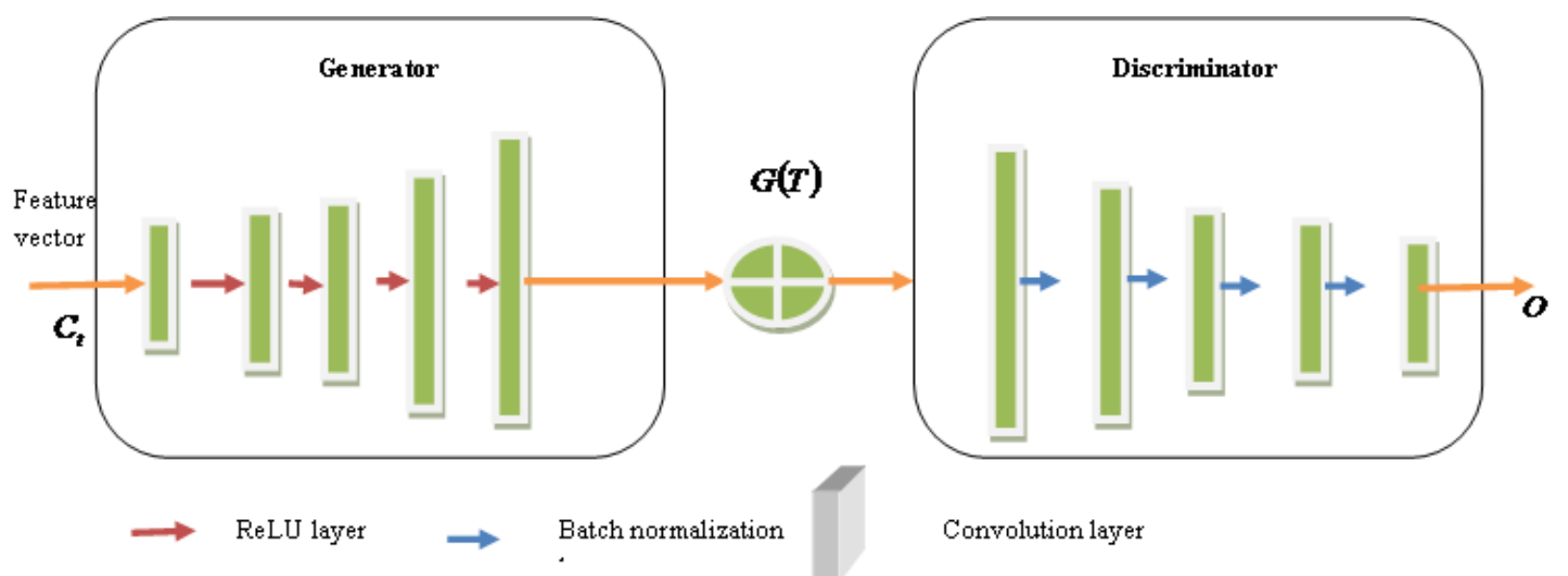

Fig.2. Block diagram of GAN

The generator, which tries to decrease the gain of the discriminator, and it is modeled as a generator loss function $\mathrm{V}_{\mathrm{g}}$ and is represented as,

$$
\mathrm{V}_{\mathrm{g}}=\max \cdot \mathrm{J}(\mathrm{Q}, \mathrm{R})
$$

The training process of GAN is developed by using the proposed ABC optimization algorithm, and hence the liver cancer detection is obtained with high accuracy.

\subsubsection{Training of GAN using ABC optimization algorithm}

In a clinical diagnosis segment, pathologists depend on the evaluation of a set of nuclei in a tissue model. The diagnostic label is utilized for tissue samples, and thus optimization technique is essential for finding sets of nuclei among cancer patients for detecting liver cancer. In this liver cancer detection method, an optimization algorithm, named ABC is developed, which is trained using the GAN classifier for creating an optimal solution with an optimal detection rate. Generally, the ABC optimization approach is applied for solving the unconstrained optimization problems, and it is a population-based stochastic method. The ABC optimization technique is an evolutionary approach, which is developed through the inspiration of intellectual foraging activities. The algorithmic procedure of the $\mathrm{ABC}$ optimization algorithm is represented below:

Step 1) Initialization: Let us assume a randomly initialized food source $F_{s}$ and the size of the food source is considered as $F_{S} \times a$. The integer values are randomly filled in a matrix ranging from 1 to $\mathrm{m}$ where the sensor nodes are employed in energy and a network is initialized to every node. The position information of every node is recognized to a sink node to perform the routing.

Step 2) Employed bee: The food sources are encoded and every element of the food source is illustrated as the index of the sensor node in the ABC algorithm. The food source of the bee is updated with the employed bee part and also the production of a new bee phase by using the below equation.

$$
\mathrm{F}_{\mathrm{x}, \mathrm{y}}^{\mathrm{i}+1}=\mathrm{F}_{\mathrm{x}, \mathrm{y}}^{\mathrm{i}}+\mathrm{U}_{\mathrm{x}, \mathrm{y}}\left(\mathrm{F}_{\mathrm{x}, \mathrm{y}}^{\mathrm{i}}-\mathrm{F}_{\mathrm{p}}, \mathrm{y}\right)
$$

Where, $F_{x, y}^{i}$ denotes the $x^{\text {th }}$ food source of $y^{\text {th }}$ value in the $i^{\text {th }}$ iteration, $U_{x, y}$ represents the random value in $[-1,1], x \in\{1,2, \ldots a\}$ and also the index of neighbor is denoted as $p, p \in\left\{1,2, \ldots, F_{S}\right\}$.

Step 3) Evaluation of fitness function: The fitness function is computed by using the difference between the actual value and estimated value. Hence, the fitness function with minimum error value is defined as an optimal solution for liver cancer detection. In addition, the fitness function is represented as,

$$
\mathrm{D}_{\mathrm{F}}=\frac{1}{\mathrm{Z}} \sum_{\mathrm{j}=1}^{\mathrm{Z}}\left(\mathrm{E}_{\mathrm{b}}-\mathrm{G}_{\mathrm{j}}\right)^{2}
$$

Step 4) Onlooker bee: In the onlooker bee phase, the food sources of the second half of the population are updated in which the food source is selected based on the below equation,

$$
\mathrm{m}_{\mathrm{x}}=\alpha_{1} \times \frac{\mathrm{A}_{\mathrm{x}}}{\max _{\mathrm{x}=1}^{\mathrm{F}_{\mathrm{s}}} \mathrm{A}_{\mathrm{x}}}+\alpha_{2}
$$


where, $\alpha_{1}$ and $\alpha_{2}$ denotes the constant and $\mathrm{A}_{\mathrm{x}}$ illustrates the fitness function applied to select the best path. The selected food source is changed with a new solution $F_{x, y}^{i+1}$. The bee seeks new food sources if the fitness $\mathrm{F}_{\mathrm{x}, \mathrm{y}}^{\mathrm{i}+1}$ is less than $\mathrm{F}_{\mathrm{x}, \mathrm{y}}^{\mathrm{i}}$, or else remains similar.

Step 5) Scout bee: If no food sources are changed for the past $W$ cycles then, these phases are executed. Furthermore, the selected food source is unused and updated by newly generated random food source.

Step 6) Termination: The abovementioned steps are continually performed until $i$ achieves the maximum cycles $\mathrm{W}_{\max }$, and the solution output is the best food source. Consequently, the communication among the base station and cluster head is executed to replace the information of sensor nodes.

\section{Results and Discussions}

The results and the discussion of a developed technique are described in this section. The experimental setup, performance metrics, dataset description, competing methods, and comparative analysis are given below.

\subsection{Experimental Setup}

The execution of the developed liver cancer detection model is performed using MATLAB with 4GB RAM, Windows 8 OS with the Intel Core i-3 processor.

\subsection{Performance Metrics}

The performance of the $\mathrm{ABC}$ optimization method is analyzed by using the three-performance metrics, such as accuracy, sensitivity, and specificity.

a) Accuracy: Accuracy is defined as the degree of closeness of the approximated value with regards to its original, and it is represented as,

$$
\text { Accuracy }=\frac{\mathrm{F}_{\mathrm{p}}+\mathrm{F}_{\mathrm{N}}}{\mathrm{F}_{\mathrm{P}}+\mathrm{T}_{\mathrm{N}}+\mathrm{F}_{\mathrm{N}}+\mathrm{T}_{\mathrm{P}}}
$$

b)Sensitivity: The ratio of positives that are accurately recognized by the classifier is, named as sensitivity and it is represented as,

$$
\text { Sensitivity }=\frac{F_{P}}{F_{P}+T_{N}}
$$

c)Specificity: Specificity is described as the ratio of negatives that are accurately recognized by the classifier and is illustrated as,

$$
\text { Specificity }=\frac{F_{N}}{F_{N}+T_{P}}
$$

where, $T_{P}$ and $F_{P}$ denotes true positive and false positive respectively, then, $T_{N}$ and $F_{N}$ illustrates the true negative and false negative respectively.

\subsection{Dataset Description}

For experimentation, the National Institutes of Health's (NIH) Clinical Center liver CT image database [23] is utilized. The NIH deep lesion database contains 32,000 annotated lesions recognized on CT images. In addition, the CT images are taken from the 4,400 different patients.

\subsection{Experimental Results}

The sample results of the developed ABC-based GAN approach for detecting liver cancer in CT images is shown in Fig. 3. 

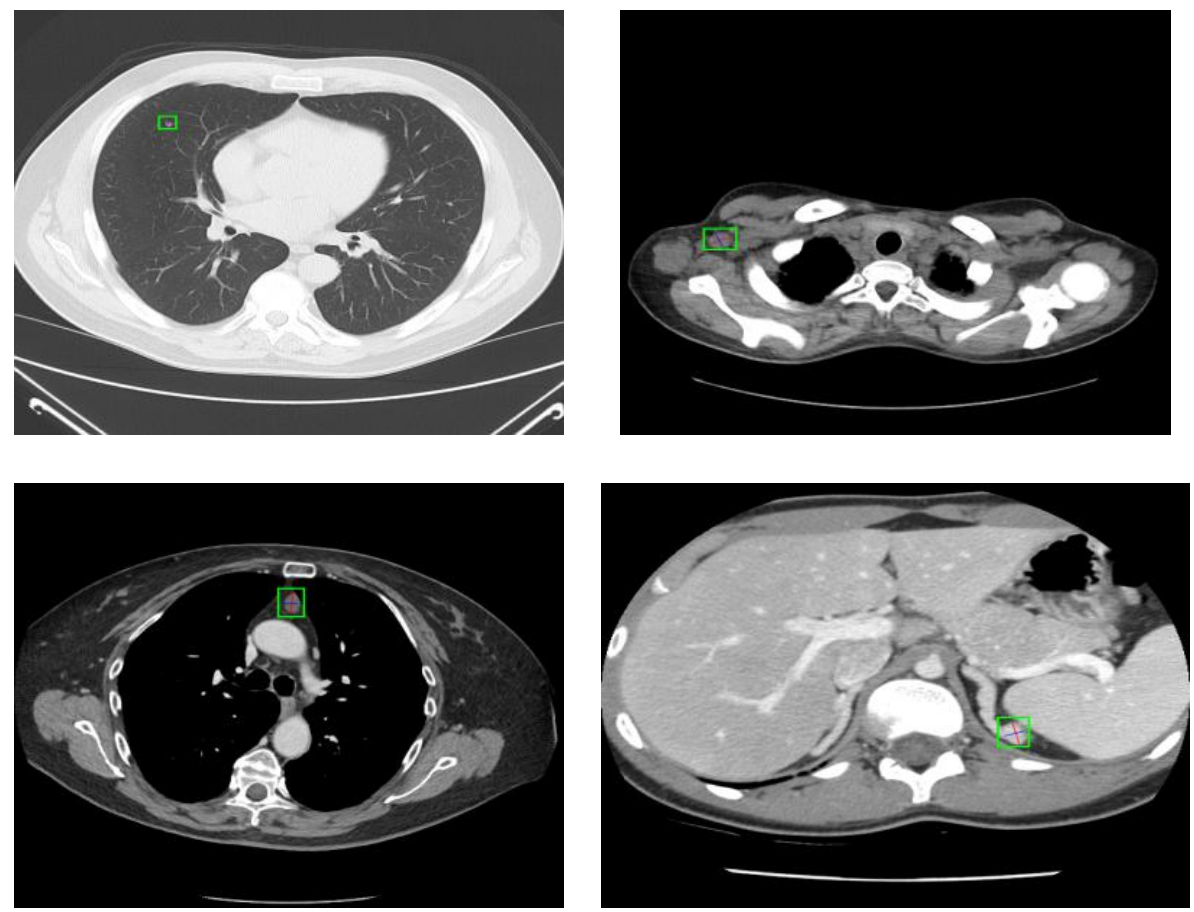

Fig.3. Sample result of the proposed liver cancer detection method

\subsection{Competing Methods}

The methods, like Deep Neural Network (DNN), random forest, K-Nearest Neighbour (KNN) are utilized for comparison with developed $\mathrm{ABC}$ algorithm for the analysis.

\subsection{Comparative Analysis}

The comparative analysis of the developed $\mathrm{ABC}$ optimization algorithm in terms of accuracy, sensitivity, and specificity parameters are computed through changing the training data percentage based on the image sizes, such as $255 * 255$ and $512 * 512$.

\subsection{Comparative Discussion}

The comparative analysis of the developed ABC-based GAN approach in terms of accuracy, sensitivity, and specificity parameters is computed by varying the training data percentage using image size $255 * 255$ and $512 * 512$.

\subsubsection{Analysis using image size $255 * 255$}

The performance analysis with respect to the accuracy, sensitivity, and specificity by changing the training data using $255 * 255$ image size is depicted in Fig. 4. The analysis based on the accuracy parameter is represented in Fig. 4a). For 90\% of training data, accuracy computed by existing Random Forest, KNN, DNN, and the proposed ABC-based GAN is $88.28 \%, 89.61 \%, 91.92 \%$, and $95.16 \%$ respectively. The analysis of schemes based on the sensitivity parameter is represented in Fig. 4b). For $90 \%$ of training data, sensitivity is estimated by the existing Random Forest, KNN, DNN, and proposed ABC-based GAN are $73.75 \%, 75.23 \%, 76.42 \%$, and $79.21 \%$. The analysis of approaches based on specificity parameter is plotted in Fig. 4c). For 90\% training data, the specificity is calculated by existing Random Forest, KNN, DNN and the proposed ABC-based GAN is $97.40 \%, 97.96 \%, 93.15 \%$, and $98.42 \%$. 


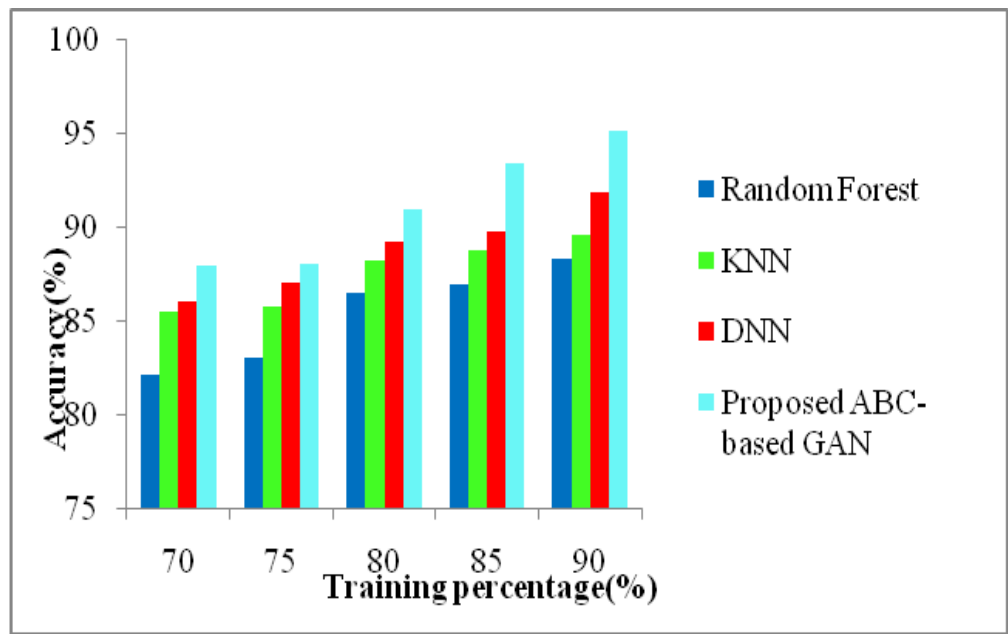

(a)

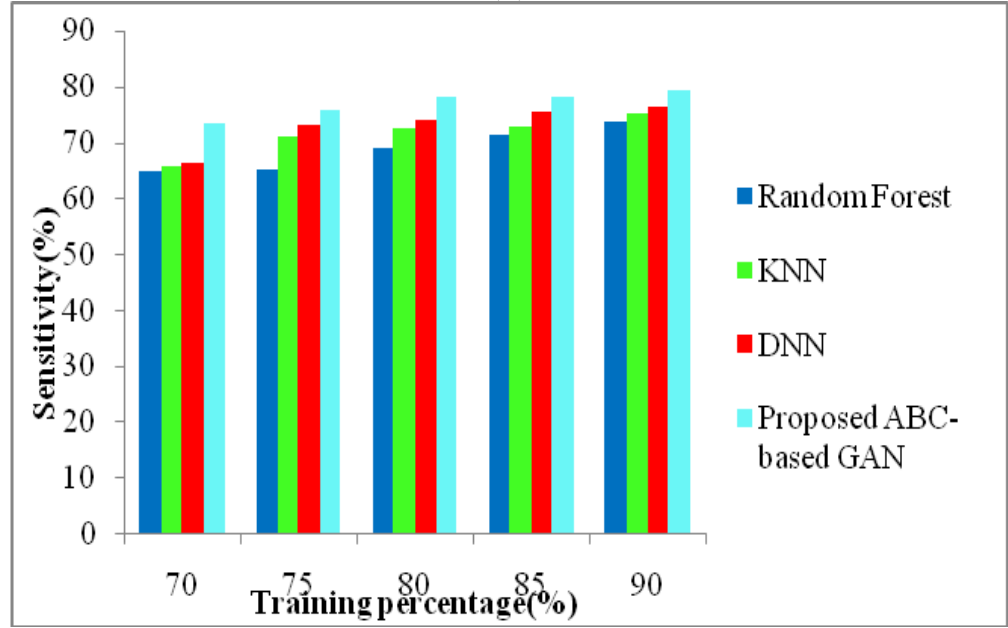

(b)

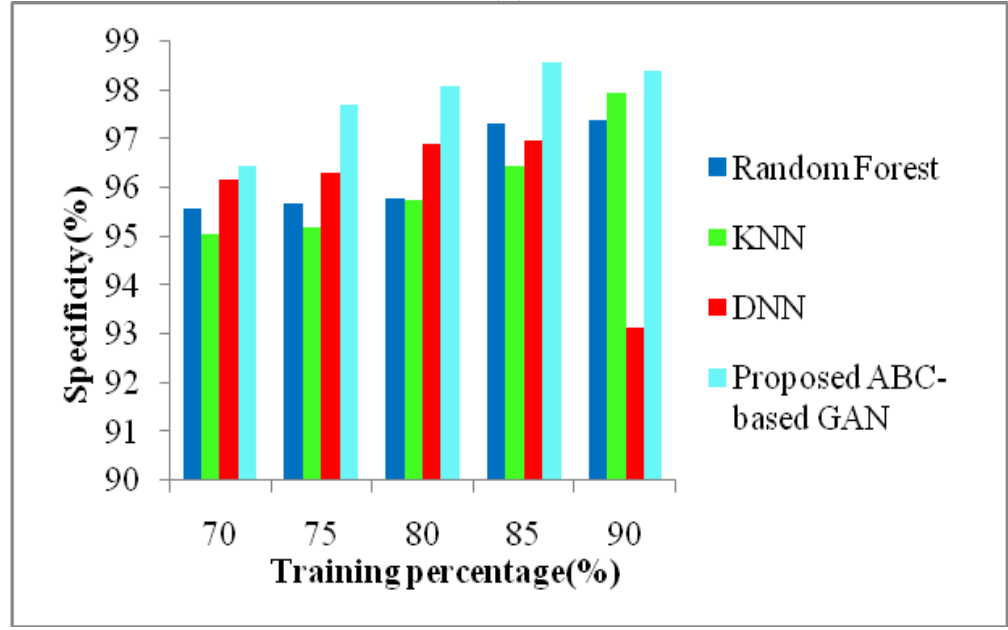

(c)

Fig.4. Analysis based on image size 255*255 a) Accuracy, b) Sensitivity, c) Specificity

\subsubsection{Analysis using image size 512 * 512}

Fig. 5 represents the analysis of the proposed method based on image size $512 * 512$ by changing training data with respect to the accuracy, sensitivity, and specificity. Fig. 5a) illustrates the analysis of various methods using the accuracy parameter. For the $90 \%$ of training data, accuracy estimated by the existing Random Forest, KNN, DNN, and the proposed ABC-based GAN is 91.93\%, 92.47\%, 92.90\%, and 93.05\%. The evaluation of existing methods based on the sensitivity parameter is shown in Fig. 5b). For $90 \%$ of the training data, sensitivity is calculated by existing Random Forest, KNN, DNN, and proposed ABC-based GAN are $89.30 \%, 90.70 \%, 91.20 \%$, and $91.88 \%$. The analysis of various techniques based on the specificity parameter is denoted in Fig. 5c). For 90\%, of training data, the specificity parameter is 
evaluated by existing Random Forest, KNN, DNN, and proposed ABC-based GAN are91.68\%, 93.86\%, $93.95 \%$, and $93.98 \%$.

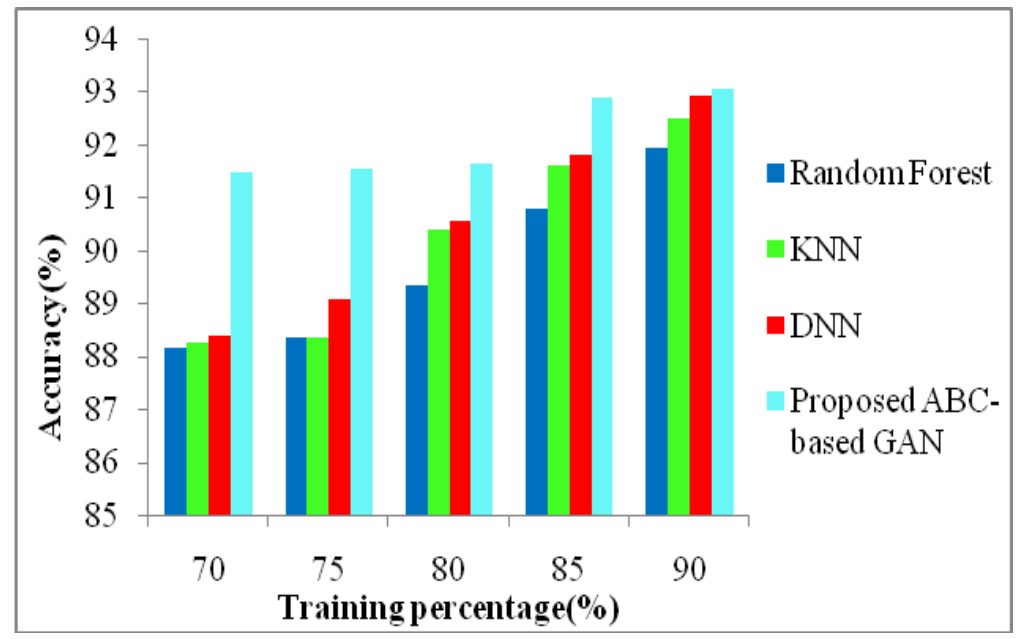

(a)

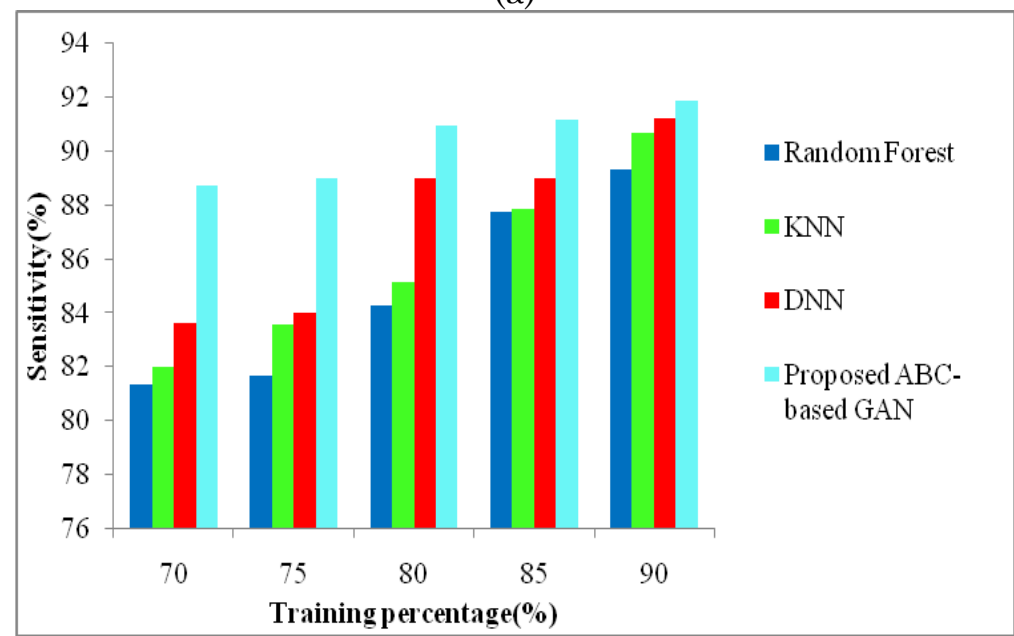

(b)

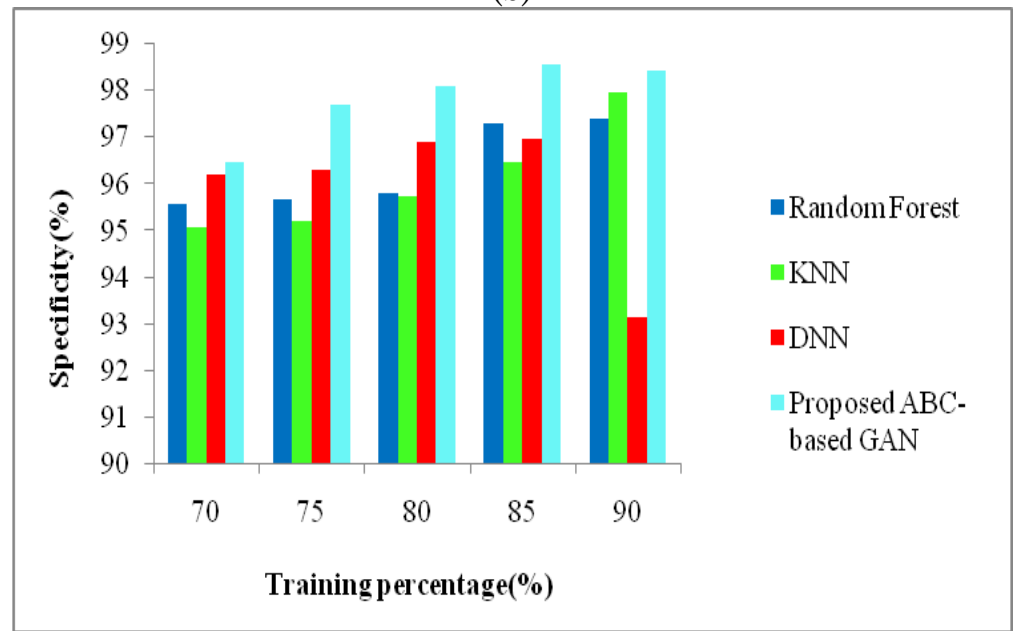

(c)

Fig.5. Analysis of various methods with training data using $512^{*} 512$ image size in terms of a) accuracy, b) Sensitivity, c) Specificity

\subsection{Comparative Discussion}

The analysis of various techniques based on training data using image sizes $255 * 255$ and $512 * 512$ in terms of accuracy, sensitivity, and specificity parameter is represented in Table 1 . The maximal accuracy of $95.16 \%$ is attained by proposed ABC-based GAN, whereas existing methods, such as Random Forest, $\mathrm{KNN}$ and DNN are $88.28 \%, 89.61 \%$, and $91.92 \%$ based on $255^{*} 255$ image size. Moreover, the maximum 
sensitivity of $91.88 \%$ is obtained by the proposed ABC-based GAN for $512{ }^{*} 512$ image size. Similarly, from comparative analysis, the developed ABC-based GAN method attains a maximum specificity value of $98.42 \%$ for the $255 * 255$ image size. From this comparative analysis table, it is well-recognized that the developed ABC-based GAN technique obtains maximum accuracy, specificity, and sensitivity.

Table1. Comparative discussion

\begin{tabular}{|cccccc}
\hline Image sizes & Metrics & $\begin{array}{c}\text { Random } \\
\text { Forest }\end{array}$ & KNN & DNN & $\begin{array}{c}\text { Proposed ABC- } \\
\text { based GAN }\end{array}$ \\
\hline $255 * 255$ & Accuracy (\%) & 88.28 & 89.61 & 91.92 & $\mathbf{9 5 . 1 6}$ \\
& Sensitivity (\%) & 73.75 & 75.23 & 76.42 & $\mathbf{7 9 . 2 1}$ \\
& Specificity (\%) & 97.40 & 97.96 & 93.15 & $\mathbf{9 8 . 4 2}$ \\
$512 * 512$ & Accuracy (\%) & 91.93 & 92.47 & 92.90 & $\mathbf{9 3 . 0 5}$ \\
& Sensitivity (\%) & 89.30 & 90.70 & 91.20 & $\mathbf{9 1 . 8 8}$ \\
& Specificity (\%) & 91.68 & 93.86 & 93.95 & $\mathbf{9 3 . 9 8}$ \\
\hline
\end{tabular}

\section{Conclusion}

In this research work, an approach, named ABC-based GAN technique is developed for liver cancer identification in CT images. At first, the liver CT images database is subjected to the liver extraction phase for liver cancer detection. After the acquisition of the input liver CT image, the liver regions are extracted from the CT image using the active contour method. Then, using the extracted liver images the cancer lesion segmentation is done. The cancer lesion regions are segmented from the liver images based on the MRF method. After the segmentation, the statistical features, such as mean, standard deviation, skewness, and kurtosis are extracted from the segmented image. Finally, the classification process is performed by means of a GAN classifier for liver cancer detection. Furthermore, the ABC optimization technique is utilized to train the developed GAN classifier. The experimentation of the developed model is performed using NIH deep lesion dataset. Moreover, the performance metrics, like accuracy, sensitivity, and specificity are evaluated and compared with other approaches. The developed ABC-based GAN technique acquired better accuracy of $95.16 \%$, maximum sensitivity, and specificity of $91.88 \%$ and $98.42 \%$, respectively. In the future, contrast enhancement will be used as a pre-processing step to further improve the detection accuracy.

\section{Compliance with Ethical Standards}

Conflicts of interest: Authors declared that they have no conflict of interest.

Human participants: The conducted research follows the ethical standards and the authors ensured that they have not conducted any studies with human participants or animals.

\section{References}

[1] Das A, Acharya U R, Panda S S, Sabut S, "Deep learning based liver cancer detection using watershed transform and Gaussian mixture model techniques", Cognitive Systems Research, vol.54, pp.165-75, May 2019.

[2] Das A, Das P, Panda S S, Sabut S, "Detection of liver cancer using modified fuzzy clustering and decision tree classifier in CT images", Pattern Recognition and Image Analysis, vol.29, no.2, pp.201-11, April 2019.

[3] [3] Hemalatha V and Sundar C, "Automatic liver cancer detection in abdominal liver images using soft optimization techniques", Journal of Ambient Intelligence and Humanized Computing, vol.6, pp.1-10, April 2020.

[4] Lakshmi Priya B, Jayanthi K, Pottakkat B, Ramkumar G, "A Modified Framework for Multislice Image Fusion for High Contrast Liver Cancer Detection.”, Journal of Research, vol.66, no.2, pp.139-49, March 2020.

[5] Xia K, Yin H, Qian P, Jiang Y, Wang S, "Liver semantic segmentation algorithm based on improved deep adversarial networks in combination of weighted loss function on abdominal CT images", IEEE Access, vol.7, pp.96349-58, July 2019.

[6] Altekruse S F, Henley S J, Cucinelli J E, McGlynn K A, "Changing hepatocellular carcinoma incidence and liver cancer mortality rates in the United States”, The American journal of gastroenterology, vol.109, no.4, pp.542, April 2014.

[7] Na K, Jeong S K, Lee M J, Cho S Y, Kim S A, Lee M J, Song S Y, Kim H, Kim K S, Lee H W, Paik Y K, "Human liver carboxylesterase 1 outperforms alpha- fetoprotein as biomarker to discriminate hepatocellular carcinoma from other liver diseases in Korean patients", International journal of cancer, vol.133, no.2, pp.408-15, July 2013. 
[8] Kim Y S, Sohn S Y, Yoon C N, "Screening test data analysis for liver disease prediction model using growth curve", Biomedicine \& pharmacotherapy, vol.57, no.10, pp.482-8, December 2003.

[9] Lu X, Xie Q, Zha Y, Wang D, "Fully automatic liver segmentation combining multi-dimensional graph cut with shape information in 3D CT images", Scientific reports, vol.8, no.1, pp.1-9, July 2018.

[10] Bartolozzi C, Ciatti S, Lucarelli E, Villari N, de Dominicis R, "Ultrasound and computer tomography in the evaluation of focal liver disease", Acta Radiologica Diagnosis, vol.22, no.5, pp.545-8, September 1981.

[11] Kononenko I, "Machine learning for medical diagnosis: history, state of the art and perspective", Artificial Intelligence in medicine, vol.23, no.1, pp.89-109, August 2001.

[12] Avşar T S and Arıca S, "Automatic segmentation of computed tomography images of liver using watershed and thresholding algorithms", In proceedings of EMBEC \& NBC 2017 Springer, pp.414-417, June 2017.

[13] Saleck M M, ElMoutaouakkil A, Mouçouf M, "Tumor detection in mammography images using fuzzy C-means and GLCM texture features", In proceedings of 14th International Conference on Computer Graphics, Imaging and Visualization, pp.122-125, May 2017.

[14] Amitha R and Jayasree M, "Automated liver detection using markov random field segmentation", Elsevier, vol.24, pp.1305 - 1310, 2016.

[15] Jiang H, Zheng R, Yi D, Zhao D, "A novel multiinstance learning approach for liver cancer recognition on abdominal CT images based on CPSO-SVM and IO", Computational and mathematical methods in medicine, January 2013.

[16] Li C, Xu C, Gui C, Fox M D, "Distance regularized level set evolution and its application to image segmentation," IEEE transactions on image processing, vol.19, no.12, pp.3243-54, August 2010.

[17] Sun C, Guo S, Zhang H, Li J, Chen M, Ma S, Jin L, Liu X, Li X, Qian X, "Automatic segmentation of liver tumors from multiphase contrast-enhanced CT images based on FCNs", Artificial intelligence in medicine, vol.83, pp.58 66, November 2017.

[18] Kass M, Witkin A, Terzopoulos D, "Snakes: Active contour models", International journal of computer vision, vol.1, no.4, pp.321-31, January 1988.

[19] Azmi R and Norozi N, "A new markov random field segmentation method for breast lesion segmentation in MR images", Journal of medical signals and sensors, no.3, pp.156, July 2011.

[20] Du Buf J H, Kardan M, Spann M, “Texture feature performance for image segmentation”, Pattern recognition, vol.23, no.3-4, pp.291-309, January 1990.

[21] Yuqing Gao, Boyuan Kong, and Khalid M. Mosalam,"Deep leaf-bootstrapping generative adversarial network for structural image data augmentation", Computer- Aided Civil and Infrastructure Engineering, vol.34, no.9, pp.755-773, 2019.

[22] Xu Y, Fan P, Yuan L, "A simple and efficient artificial bee colony algorithm", Mathematical Problems in Engineering, Article ID 526315, DOI: 10.1155/2013/526315, January 2013.

[23] National Institutes of Health's (NIH) Clinical Center liver CT image database taken from, "https://nihcc.app.box.com/v/DeepLesion/folder/50715173939", accessed on July 2020.

[24] Yogita Ashok Deore and Namrata D. Ghuse, "Efficient Image Processing Based Liver Cancer Detection Method," International Journal on Emerging Trends in Technology, vol. 3, no. 3, 2020.

[25] Siqi Zhang, Jiaxi Cheng, Wei Shi, Kai-Bin Li, De-Man Han, and Jing-Juan Xu, "Fabrication of a Biomimetic Nanochannel Logic Platform and Its Applications in the Intelligent Detection of miRNA Related to Liver Cancer," American Chemical Society, vol. 92, no. 8, pp. 5952-5959, 2020. 